\title{
Y a-t-il des mots des montagnards? Le lexique et les choix politiques montagnards
}

Was there a Montagnard vocabulary? The lexicon and the political choices of the Montagnards compared with other deputies

\section{Marco Marin}

\section{(2) OpenEdition}

\section{Journals}

Édition électronique

URL : https://journals.openedition.org/ahrf/13611

DOI : 10.4000/ahrf.13611

ISSN : 1952-403X

Éditeur :

Armand Colin, Société des études robespierristes

Édition imprimée

Date de publication : 1 septembre 2015

Pagination : 55-76

ISBN : 9782200929855

ISSN : 0003-4436

Référence électronique

Marco Marin, "Y a-t-il des mots des montagnards ? Le lexique et les choix politiques montagnards », Annales historiques de la Révolution française [En ligne], 381 | juillet-septembre 2015, mis en ligne le 01 septembre 2018, consulté le 22 avril 2022. URL : http://journals.openedition.org/ahrf/13611 ; DOI :

https://doi.org/10.4000/ahrf.13611 


\title{
Y A-T-IL DES MOTS DES MONTAGNARDS? LE LEXIQUE ET LES CHOIX POLITIQUES MONTAGNARDS
}

Marco MARIN

\begin{abstract}
Dans notre article, nous analysons le rapport entre les choix et actions politiques et le discours, en considérant le discours luimême comme une action. Nous focalisons d'abord notre attention sur l'histoire des désignants politiques « Montagne », "Gironde » et " sans-culottes ", qui nous paraissent relever d'un système complexe d'hétéro-désignation et d'auto-désignation. Une fois acceptés, ces mots sont employés par tous les acteurs politiques. II en va de même, d'ailleurs, de certaines notions comme l'apologie de la «pauvreté » et de la « médiocrité », qui ne sont pas exclusivement le fait de la Montagne. Pour autant, cette dernière se distingue par l'usage de certaines expressions, comme le démontre son invitation à « républicaniser le commerce ».
\end{abstract}

Mots-clés : Montagne, Gironde, sans-culottes, pauvreté, médiocrité, " républicaniser le commerce »

Saisir les spécificités discursives des montagnards par rapport à celles des autres membres de la Convention n'est pas une tâche aisée, puisque une grande partie du lexique politique est commune à tous les députés de l'Assemblée. Afin de mener une étude des usages discursifs des députés à la Convention, il serait nécessaire d'avoir à disposition de grands corpora - comme le texte complet des Archives parlementaires ${ }^{1}$ ou du Moniteur Universel - pour les analyser avec les logiciels lexicométriques. Avec ces outils, on pourrait comparer, par exemple, l'usage que les divers acteurs

(1) Archives Parlementaires (désormais AP), 102 vol., Paris, Dupont/CNRS, 1862-2012. 
font de certaines lexies ${ }^{2}$ par des listes d'occurrences, de co-occurrences et par des diagrammes temporels.

Faute de ces outils, nous présenterons dans cet article un cadre général du lexique montagnard, notamment celui de quelques personnages affiliés à la gauche parlementaire, en ayant recours à une perspective comparative avec les autres groupes de l'Assemblée, les girondins en particulier ${ }^{3}$. Cette approche nous est indispensable pour saisir les éventuelles différences ou similitudes discursives des divers groupes, qui contribuent à guider leur action et fondent leur identité politique. Grâce au matériel dont nous disposons, nous examinerons surtout les textes des trois montagnards, parmi les plus connus - Robespierre, Marat et Saint-Just -, en utilisant aussi les discours issus des deux cent soixante-quatre autres députés de gauche recensés par Françoise Brunel ${ }^{4}$.

Le corpus des sources analysées est assez vaste. Tout d'abord, il y a les corpora numériques que nous avons créés à l'Université de Trieste. Il s'agit des Euvres de Robespierre; des Euvres complètes de SaintJust ; des CEuvres politiques de Marat; du Père Duchesne d'Hébert ${ }^{5}$. Nous y ajouterons quarante-cinq discours de Danton publiés par Hector Fleischmann ${ }^{6}$. Ces sources ont été analysées à travers des logiciels de lexicométrie ${ }^{7}$. Nous avons également pris en considération les discours

(2) Le terme lexie a été introduit par le linguiste Bernard Pottier (Bernard PoTTIER, Systématique des éléments de relation, Paris, Klincksieck, 1962 ; Idem, Linguistique générale, théorie et description, Paris, Klincksieck, 1974). Il est utilisé pour définir « toute unité lexicale mémorisée en langue » et peut être employé, soit pour définir un seul mot, soit pour indiquer un groupe de mots, s'il a une unité de signification. Par exemple le mot « droit » est une lexie, mais « droit du citoyen » est une lexie également.

(3) Rappelons que les girondins et les montagnards sont des courants politiques aux contours flous et fluctuants. À ce propos, Augustin CHALlAMEL, Les clubs contre-révolutionnaires, cercles, comités, sociétés, salons, réunions, cafés, restaurants et librairies, Paris, Leopold Cerf, 1895, p. 471 et $s q$; Albert SoBoul (dir.), Girondins et Montagnards, Paris, SER, 1980 ; François FuRET, Mona Ozouf (dir.), La Gironde et les Girondins, Paris, Payot, 1991.

(4) Françoise Brunel, Les députés montagnards, dans Albert Soboul (dir.), Girondins et Montagnards, op. cit., p. 343-361 ; Idem, « Montagnards/Montagne », dans Albert Soboul (dir.), Dictionnaire historique de la Révolution française, Paris, PUF, 2005 (1 ${ }^{\text {re }}$ éd. 1989), p. 757-761. Le recensement de Françoise Brunel se réfère à juin 1793. Voir aussi, Michael S. LEWIS-BECK, Anne HILDRETH, Alan B. SPITZER, « Y a-t-il eu un groupe girondin à la Convention nationale (1792-1793) ? », dans François Furet, Mona Ozouf (dir.), La Gironde et les Girondins, op. cit., p. 169-188. Ces derniers reprennent, à travers une analyse statistique des six appels nominaux entre janvier et mai 1793, les questions de l'existence de deux groupes organisés de girondins et montagnards au sein de la Convention, en concluant que, lors du procès au roi, il était déjà possible de distinguer un groupe montagnard, tandis qu'un groupe girondin, assez homogène, se forme seulement entre avril et mai 1793.

(5) Cf. Cesare Vetter, Marco MARIN (dir.), La felicità è un'idea nuova in Europa, tome 2, Trieste, EUT, 2013.

(6) Hector Fleischmann (dir.), Discours civiques de Danton, Paris, E. Fasquelle, 1920.

(7) Nous avons utilisé principalement les logiciels Cordordance et Lexico 3. 
de plusieurs autres députés, de manière à vérifier l'usage et la fréquence de certaines lexies. Ici, nous nous sommes référés au portail ARTFL de l'Université de Chicago où se trouvent les quatre-vingt-deux premiers volumes des Archives parlementaires ${ }^{8}$. Enfin, sans recours aux outils informatiques, nous avons repris le texte du Moniteur universel et le recueil des sources La société des Jacobins d'Aulard", afin de pouvoir associer une lecture plus classique aux indications issues du traitement automatique des autres données.

\section{Définir et choisir son camp : les désignants socio-politiques}

\section{La définition d'un groupe : "Montagne », de hétéro-désignant à auto-désignant et vice versa?}

En général, on considère que la première occurrence de " Montagne » dans les débats à l'Assemblée remonte à la séance du 27 octobre $1791^{10}$, lorsque Lequinio prononce la phrase : "Citoyens français, vous nous avez honorés de votre confiance, et votre estime nous a portés sur le haut de la montagne d'où nos regards s'étendent sur tout le royaume $»^{11}$. Or, il faut noter que dans ce discours l'auteur n'emploie pas le terme en tant que désignant des députés de la gauche, mais plutôt pour se référer à l'ensemble de l'Assemblée législative. Lequinio, donc, n'évoque pas la « Montagne », dans le sens qui s'est affirmé historiquement. Pour cette raison, il nous semble préférable d'indiquer comme première occurrence de « Montagne » dans le sens qu'elle a acquis pendant la Révolution, celle présente dans le débat à l'Assemblée du 21 mars 1792, publié dans le tome 40 (p. 217) des Archives parlementaires : « Un membre du côté droit, placé à la droite de M. Calvet : "Monsieur le président, je demande que vous rappeliez à l'ordre Monsieur (il désigne un membre placé à la gauche de M. Calvet) qui m'a dit que je n'étais pas ici à ma place, que je serais mieux sur la Montagne" ».

(8) Ces volumes contiennent toutes les séances des assemblées jusqu'au 4 janvier 1794. Soulignons que le portail ARTFL ne peut pas être considéré comme une source exhaustive par rapport aux textes des $A P$, vu la grande quantité d'erreurs produite par l'OCR. Voir http://artfl-project.uchicago. edu/node/144.

(9) Àiphonse ÁulARD, La société des Jacobins, Paris, Quantin, 1892, 6 voi.

(10) Albert MATHIEZ, Les origines des cultes révolutionnaires, Paris, Soc. Nouvelle de Librairie et d'édition, 1904, p. 91 ; Françoise BRUNEL, "Montagnards/Montagne », op. cit. ; Claire GASPARD, «Le thème de la Montagne », dans Langages de la Révolution (1770-1815), Paris, Klincksieck, 1995, p. 329-336.

(11) $A P$, t. 34 , p. 441 
En rapprochant cette occurrence d'un propos assez connu de Robespierre, mais plus tardif ${ }^{12}$, nous avons l'impression que « Montagne », dans le sens qui nous intéresse, aurait pu apparaître à l'origine comme un hétéro-désignant ${ }^{13}$. Il ne s'agirait donc pas d'un auto-désignant comme on pourrait le croire en suivant Lequinio. Lisons le passage de Robespierre : « [Duhem] est appuyé, avec vigueur, par cette partie de l'Assemblée qui siège ordinairement dans le coin de la salle qu'on nomme vulgairement la Montagne $»^{14}$. Si notre intuition est fondée - mais, à ce stade, de plus amples approfondissements sur la période octobre 1791-juillet 1792 seraient nécessaires - l'hypothèse serait de rattacher l'origine de ce désignant à la fameuse fable La montagne accouchant d'une souris, rendue célèbre au $\mathrm{XVII}^{\mathrm{e}}$ siècle par La Fontaine ${ }^{15}$ et qui reste très populaire à la fin du XVIII ${ }^{\mathrm{e} 16}$. Selon cette version, les montagnards seraient ceux qui, avec beaucoup de fracas, promettent de grandes réformes mais ne parviennent pas à les faire aboutir.

En suivant Alexandre Guermazi ${ }^{17}$, il est cependant nécessaire d'indiquer que, au moins à partir de l'été 1792, le lemme « Montagne » est employé comme auto-désignant, notamment par Chabot le 18 juillet 1792 aux Jacobins ${ }^{18}$. Toutefois, en opérant aussi un recensement des occurrences, Guermazi spécifie que ce n'est qu'à partir du mois de décembre 1792, et lors du débat sur le procès au roi, que ce terme « est utilisé lorsque

(12) Signalons que nos recherches indiquent que Robespierre accepte tardivement l'introduction des nouvelles expressions dans son vocabulaire ou le changement de sens de vieux mots. Par rapport à Marat, par exemple, il utilise plus tard « sans-culottes » et « démocratie », ainsi qu'il accepte tard la synonymie « démocratie »-« république » (déjà proposée par Desmoulins, par exemple, dans le $1^{\mathrm{er}}$ numéro des Révolutions de France et de Brabant du 28 novembre 1789). À ce propos, Annie Geffroy affirme : « Notons [...] le "retard énonciatif" de Robespierre : en février, il recule encore devant l'emploi de jacobin; en oct.-nov., il utilisera encore sans-culottes comme une citation, un mot de "l'autre camp" »; voir : « Sans-culotte(s). novembre 1790-juin 1792 », dans Dictionnaire des usages socio-politiques (1770-1815), fasc. 1, Paris, Klincksieck, 1985, p. 172.

(13) Sur les définitions d'auto et d'hétéro-désignant, voir Annie GEFFROY, « Le nous de Robespierre ou le territoire impossible », Mots, mars 1985, p. 63.

(14) Maximilien RoBESPIERRE, Lettres à ses commettants, ${ }^{\circ} 9$ (14 déc. 1792), dans idem, Euvres (désormais $O M R$ ), 11 tomes, Paris, SER, 1910-2007, t. 5, p. 139. Pour les autres occurrences de « Montagne » pendant le printemps 1792, voir AP, t. 42, p. 431, 739.

(15) Voici les vers de La Fontaine: «La Montagne qui accouche ». Une Montagne en mal d'enfant | Jetait une clameur si haute, | Que chacun au bruit accourant | Crut qu'elle accoucherait, sans faute, | D'une Cité plus grosse que Paris : | Elle accoucha d'une Souris. | Quand je songe à cette Fable | Dont le récit est menteur | Et le sens est véritable, | Je me figure un Auteur | Qui dit : Je chanterai la guerre | Que firent les Titans au Maître du tonnerre. | C'est promettre beaucoup : mais qu'en sort-il souvent? | Du vent ».

(16) Pour ce qui concerne nos sources nous avons retrouvé l'expression chez Marat et Hébert. On retrouve certaines occurrences de ce dicton dans les $A P$.

(17) Cf. article supra.

(18) Alphonse AUlARD, La société des Jacobins, op. cit., t. 4, p. 114. 
les jacobins appellent à se mobiliser pour faire valoir à la Convention les orientations prises publiquement au sein du Club».

En renvoyant à l'article d'Alexandre Guermazi pour un approfondissement de ces questions, il est toutefois possible d'affirmer que « Montagne » et « montagnard(s) » sont de plus en plus utilisés en 1793, en devenant, au fur et à mesure, des lexies ayant une valeur axiologique tout à fait méliorative. Pour ce qui concerne nos trois montagnards de référence, tous commencent à utiliser la lexie « Montagne » entre décembre et janvier 1792-1793. Marat et Robespierre l'emploient régulièrement, tandis que pour Saint-Just l'Allocution aux Jacobins du $1^{\text {er }}$ janvier 1793 est le seul témoignage de cet usage jusqu'en septembre suivant. Par ailleurs, il n'est pas négligeable qu'elle soit utilisée dans une logique d'opposition avec « la faction brissotine»: «Citoyens, vous n'ignorez pas que, pour dissiper les erreurs dont Roland a couvert toute la république, la Société a arrêté qu'elle ferait imprimer et distribuer le discours de Robespierre. Nous l'avons regardé comme une éternelle leçon pour le peuple français, comme un sûr moyen de démasquer la faction brissotine et d'ouvrir les yeux des Français sur les vertus trop longtemps inconnues de la minorité qui siège sur la Montagne [écrit Saint-Just] $»^{19}$.

Lors du procès au roi, puis lors de l'affrontement entre la Montagne et la Gironde (de janvier à mai 1793), l'usage axiologique et hagiographique de «Montagne », qui fait également plusieurs renvois à la Bible et à la Nature rousseauiste ${ }^{20}$, permet aux députés de gauche de trouver une unité en tant que groupe politique, tant par rapport aux membres qui le composent que par rapport à ceux qui lui sont extérieurs. Néanmoins, ce n'est qu'après les journées du 31 mai- 2 juin que ce terme est introduit progressivement dans le lexique politique commun, en surmontant aussi les résistances de certains conventionnels. Un exemple de ces réticences est la tentative désespérée et tardive d'un député de la Plaine, Barthélémy Albouys, qui, à la fin juin 1793, tente de neutraliser la force identificatrice et évocatoire de la lexie «Montagne », en la décrivant comme dangereuse pour l'égalité entre les députés et entre les citoyens :

« Si nous sommes tous égaux entre nous, [...] d'où vient cette distinction de côté droit et de côté gauche, de montagne, de plaine et de marais ? [...] Montagnards, ne vous effrayez pas : je n'introduis point le levier dans les fentes de vos rochers ; je gonfle les eaux de ce marais, j'en couvre la

(19) Louis-Antoine de SAINT-Just, Euvres complètes, Paris, G. Lebovici, 1984, p. 402.

(20) Cf. Claire GASPARD, «Le thème de la Montagne », op. cit. 
plaine, et tous nous nous élèverons au plus haut du mont sacré : là sans doute est un terrain uni, où tous au même niveau, nous ne connaîtrons plus aucune distinction de places. [...] Puisque le nom de montagne présage encore à la France une distinction d'ordres, prenons tous, dès aujourd'hui, le titre de députés à la montagne, et que ce nom cesse d'être terrible en devenant celui de l'entière image du peuple souverain $»^{21}$.

Si pendant l'an II, la lexie «Montagne », tout comme «sansculotte(s) », fait partie intégrante du langage officiel de la République, le 9 thermidor n'en décrète pas le bannissement immédiat, ce qui montre qu'elle n'était pas un synonyme de « robespierristes », mais qu'elle était plutôt associée aux institutions républicaines. Un exemple parmi d'autres est celui du comité de surveillance de Rethel-sur-Aisne qui, le 15 thermidor an II, termine sa lettre de félicitations pour la faillite de la « conjuration » de Robespierre avec : «Vive la Convention! Vive la montagne ! Vive la République ! $»^{22}$.

Néanmoins, après la chute des robespierristes, la valeur positive du lemme «Montagne » ne dure pas longtemps. Dans les pétitions des sociétés populaires et des autorités constituées les expressions «Vive la Montagne », « braves montagnards », etc. cessent d'être employées dès la première quinzaine de vendémiaire an III $^{23}$. Dans une lettre du 24 frimaire an III (14 décembre 1794), le député Pierre-Amable Soubrany peut affirmer « qu'il suffit de demander la parole du haut de la Montagne pour être écarté par l'ordre du jour $»^{24}$. Bientôt, à la suite de la fermeture du club des Jacobins ${ }^{25}$, «Montagne » devient (ou redevient) surtout un hétérodésignant, bien que les insurgés de Prairial an III crient encore «Vive la Montagne » avant d'être dispersés ${ }^{26}$.

\section{Comment nommer l'adversaire politique : " girondins », «brissotins », « rolandins » ou « buzotins »?}

Il y a vingt-cinq ans déjà que Marcel Dorigny affirmait, dans le Dictionnaire dirigé par Albert Soboul : « Le mot "girondins", pour désigner

(21) $A P$, t. 67, p. 176.

(22) $A P$, t. 94 , p. 526

(23) Françoise BRUNEL, «Les derniers Montagnards et l'unité révolutionnaire », AHRF, n 229, 1977, p. 388 , n. 15.

(24) Pierre-Auguste Soubrany, Dix-neuf lettres, Clermont-Ferrand, Boucart, 1867, p. 44. Lettre à Debreuil, du 24 frimaire an III.

(25) Aux Jacobins, « montagnards » est prononcé pour la dernière fois lors de l'avant-dernière séance du 10 novembre 1794, par Pérès.

(26) Françoise BRUNEL, « Les derniers Montagnards », op. cit., p. 388. 
un groupe politique de la Convention, s'imposa seulement au cours du $\mathrm{XIX}^{\mathrm{e}}$ siècle ; il avait été utilisé dès l'Assemblée législative, mais on lui préférait alors les expressions de Brissotins, Rolandins ou Buzotins $»^{27}$.

La consultation des corpora numériques désormais disponibles permet de préciser que, si le substantif/adjectif « brissotin(e)(s) » semble être le désignant le plus employé (plus que « girondin(e)(s)»), cela ne semble cependant pas être le cas pour « $\operatorname{rolandin}(\mathrm{e})(\mathrm{s}) »(\mathrm{et} \ll \operatorname{rolandist}(\mathrm{e})(\mathrm{s}) »)$, et encore moins pour «buzotin(e)(s)», qui est aussi rare que «louve$\operatorname{tin}(\mathrm{e})(\mathrm{s}) »$. Ainsi, chez Robespierre et Saint-Just, la formule «brissoti* » est utilisée deux fois plus que $«$ girondi* ${ }^{28}$. En outre, chez Robespierre, le terme « rolandi* » paraît 5 fois. Ce n'est pas le cas pour «buzoti* », qui n'apparaît jamais, ni chez Saint-Just, d'ailleurs. Chez Marat, nous avons 123 occurrences de « brissoti* », 23 de « girondi $*$ », 49 de « rolandi* » et 10 de « buzoti*».

Signalons encore que, pour ces auteurs, c'est la formule « brissoti* » qui s'impose d'abord. Dans Marat, elle apparaît déjà dans un Placard du 28 août 1792, où l'Ami du peuple parle d'une « faction brissotine ». En novembre, cette fois, Marat introduit l'offense : « clique brissotine ou rolandine », tandis que Robespierre commence à employer « brissoti* ». « Girondi* », cependant, ne fait son apparition dans le lexique maratiste et robespierriste qu'en janvier $1793^{29}$.

Les données issues du portail de l'ARTFL ${ }^{30}$ sont partiellement différentes. Ici aussi, l'emploi de « buzotin(e)(s) » est presque négligeable, mais sont très peu utilisées aussi les formules « rolandin(e)(s)/rolandist (e)(s) ». En revanche, les formes « brissoti* » et « girondin*/girondist* » sont très fréquentes et leur incidence est comparable. En outre, ces occurrences apparaissent souvent ensemble, comme dans le discours de Vergniaud du 13 mars 1793 : « Depuis quelques jours surtout ils y criaient avec fureur que le seul reproche qu'on pût faire aux journées de septembre c'était d'avoir été incomplètes ; qu'il fallait purger la terre du conseil exécutif des généraux, des Brissotins, des Girondins, des Rolandins, de tous ceux, en un mot, qu'ils avaient inscrits sur leurs listes de proscriptions $»^{31}$.

(27) Marcel DORIGNY, «Gironde/Girondins », dans Albert Soboul (dir.), Dictionnaire historique, op. cit., p. 503.

(28) Occurrences : Robespierre : «brissoti*», 69 ; « girondi*», 35 ; Saint-Just : 5 et 3.

(29) Signalons une pétition des habitants du département de la Sarthe, qui se plaignent des « factions maritistes [sic] et girondines, qui ne tendent qu'à détruire la République » $(A P$, t. 57, p. 376).

(30) En considérant le fort pourcentage d'erreurs dans le texte, les résultats ne sont pas totalement fiables. Cependant, nous croyons que les rapports entre les fréquences des diverses lexies ne s'éloignent pas du résultat obtenu.

(31) $A P$, t. 60, p. 163. 
Comme le montre bien cette citation, tous ces termes sont employés en tant qu'hétero-désignants, principalement par les montagnards, dans l'Assemblée (et aux Jacobins); jamais avant le 10 août et surtout après les journées du 31 mai-2 juin. À la suite de l'épuration des girondins, en effet, toutes ces formes entrent à part entière dans le groupe d'expressions utilisées pour indiquer, génériquement, l'ennemi intérieur. Elles deviennent, de la même manière que « contre-révolutionnaire(s) », « aristocrate(s) », « vicieux », etc., axiologisées en mal jusqu'à la réhabilitation et la réintégration des girondins survivants au cours de l'an III. Pendant une courte période, en mai-juin 1793, seule la forme « girondi* » devient un auto-désignant politique. On remarque cela, notamment, dans le Patriote français de Brissot du 6 mai 1793 ; et, d'une manière bien plus affirmée, dans la très connue Lettre de la Société républicaine de Bordeaux à la Convention nationale du 8 mai 1793 : « Oui, nous sommes tous Girondins ; nous le serons jusqu'au tombeau $»^{32}$.

\section{Les alliés populaires : les « sans-culotte(s) »}

La lexie « sans-culotte(s) » et les variantes « $\operatorname{san}(\mathrm{s}) \operatorname{culot}(\mathrm{t}) \mathrm{e}(\mathrm{s})$ » et « sans culot(t)e(s) » apparaissent dans les salons du XVIII ${ }^{\mathrm{e}}$ siècle comme une plaisanterie pour désigner les lettrés n'ayant pas de protection. Après un usage initial diffamatoire de la part des publicistes anti-révolutionnaires (surtout de François Marchant) ${ }^{33}$, cette lexie s'affirme progressivement avec une connotation positive au cours de la Révolution à partir de la période janvier-août $1792^{34}$. Hébert l'emploie deux fois en novembre 1790 et en février 1791, pour ensuite l'intégrer dans son lexique l'année suivante. À partir d'avril 1792, il l'emploie même plus souvent que la lexie

(32) $A P$, t. 65, p. 686 .

(33) Marchant est le rédacteur du journal Sabbats Jacobites.

(34) Pour ce qui concerne l'usage de la lexie « Sans-culotte(s) », voir Annie GEFFROY, « Sansculotte(s) (novembre 1790-juin 1792) », op. cit. ; Idem, «Désignation, dénégation : la légende des sans-culottes (1780-1980) », dans Christian CROISILLE, Jean EHRARD, Marie-Claude CHEMIN (dir.), La légende de la Révolution, Clermont-Ferrand, Univ. Blaise Pascal, 1988, p. 581-592 ; Michael SONENSCHER, Sans-Culottes : An Eighteenth-Century Emblem in the French Revolution, Princeton (NJ), Univ. Press, 2008. Pour ce qui concerne la réalité historique de la « sans-culotterie », voir Albert SOBOUL, Les sans-culottes parisiens en l'an II. Mouvement populaire et gouvernement révolutionnaire (2 juin 1793-9 thermidor an II), Paris, 1958. Sur le mouvement sans-culotte et sa définition en termes historiographiques, voir aussi Michel VOVELLE, «Le Sans-culotte marseillais », Histoire \& Mesure, vol. 1, n 1, 1986, p. 75-95; Haïm BURSTIN, « I sanculotti : un dossier da riaprire », Passato e presente, $\mathrm{n}^{\circ} 10,1986$, p. 23-52 ; Idem, La politica alla prova. Appunti sulla Rivoluzione francese, Milano, Francoangeli, 1989, p. 139-180 ; Id., L'invention du sans-culotte. Regards sur Paris révolutionnaire, Paris, Odile Jacob, 2005. Pour expliquer ce processus logique et discursif, Annie GEFFROY (« Sansculotte(s) », op. cit., p. 172) utilise l'expression « retournement énonciatif »: « Ce qui était injure dans la bouche de l'énonciateur initial disqualifié par les événements politiques devient titre de gloire ». 
« citoyens $»^{35}$. Desmoulins l'introduit de temps en temps dans son lexique à partir d'octobre 1791 .

Pour ce qui concerne nos trois montagnards, Marat emploie la lexie «sans-culotte(s) » à partir de juillet 1792, avec une fréquence de plus en plus importante jusqu'en juillet 1793. Dans le corpus de SaintJust, elle apparaît bien plus tard et très rarement : en deux occasions dans des documents collégiaux et une seule fois dans un rapport écrit individuellement ${ }^{36}$. Robespierre, quant à lui, l'utilise un peu moins d'une centaine de fois, principalement pendant l'année 1793 - et seulement deux fois au singulier en $1794^{37}$. En regardant la liste des concordances chez ce dernier auteur, il est surprenant de voir comment une des premières occurrences de « sans-culottes » est déclinée le 10 mai 1792, à savoir à côté de deux lexies ayant une valeur fortement dépréciative : «Je m'attends bien que je serai dénoncé par ses auteurs, par tous les ennemis de la liberté, comme le défenseur de l'anarchie, des sans-culottes, des perturbateurs ${ }^{38}$. Les éléments ici évoqués pourraient être interprétés comme des indices de l'aversion de Robespierre pour ce désignant. En effet, il l'emploie surtout dans la période mai-juillet 1793, à savoir lors de l'affrontement le plus dur avec les girondins.

Aux Jacobins, «sans-culotte(s) » est introduit par Desmoulins et Pierre-François Réal à la fin de 1791 et devient plus courant pour certains membres (Chabot) à l'été 1792. Ici, pendant l'an II, il apparaît chez Robespierre et dans les discours d'Albitte, Couthon, Collot-d'Herbois, Legendre, Barère, Fouché, Dumas et Dechamps ${ }^{39}$.

À partir du portail ARTFL, on peut souligner que la première occurrence de « sans-culottes » dans les Archives parlementaires apparaît dans une Lettre de M. Faydel à ses commettants de fin septembre $1791^{40}$.

(35) Voir Marco MARIN, «Citoyenneté, République, Démocratie : une approche quantitative », Révolution Française.net, mai 2015, http://revolution-francaise.net/2015/05/01/614-citoyenneterepublique-democratie-une-approche-quantitative.

(36) Dans le Rapport [...] sur les trente-deux membres de la Convention détenus en vertu du décret du 2 juin (8 juillet 1793), dans Louis-Antoine SAINT-JUST, Euvres complètes, op. cit., p. 457 et $s q$.

(37) Cela probablement pour prendre ses distances et se différencier des hébertistes, devenus entre temps des adversaires politiques.

(38) Voir OMR, t. 8, p. 346. Le passage est repris de cette manière dans le Journal des débats et de correspondance de la Société des Amis de la Constitution, $\mathrm{n}^{\circ} 193$, p. 2. Le Journal général de France $\left(\mathrm{n}^{\circ} 136\right.$, p. 543$)$ fournit une version très similaire : « Je m'attends bien que je serai dénoncé par les auteurs de la liste civile, par tous les ennemis de la liberté, comme le défenseur de l'anarchie, des sans-culottes ».

(39) Les vérifications ont été effectuées avec un système automatique sur le $6^{\mathrm{e}}$ vol. d'Alphonse AUlARD, La société des Jacobins, op. cit.

(40) AP, t. 32, p. 469 
Toutefois, l'auteur ne semble pas encore l'employer dans le sens que l'expression a historiquement acquis. Pour notre propos, le passage du montagnard et jacobin Bréval issu du discours non prononcé Sur l'affaire d'Avignon de mars 1792 est plus utile :

« On appelle [...] le peuple français d'Aix sans-culottes, mais on devrait dire qu'il aurait des culottes et du pain, si la liste civile n'était pas aussi énorme ; mais il a du courage et des piques, et il maintiendra la Constitution et sa liberté. Pasquier, dans ses recherches sur la France, dit que les brigands étaient les premières troupes de l'Empire, $[\ldots]$ ces derniers $[\ldots]$ ont été remplacés par les sans-culottes, c'est-à-dire par la vertu $\gg^{41}$.

Même si ce discours n'a pas été prononcé, il est important de le signaler, car il fait remonter en arrière l'introduction de la lexie dans l'espace de l'Assemblée. Par ailleurs, quelques jours plus tard, le 11 mars, le ministre de l'Intérieur, Cahier de Gerville, emploie lui aussi «sansculotte » à l'Assemblée en tant que désignant social : "On a remarqué sous le déguisement de sans-culottes des hommes qui portaient du linge fin, et qui, par leur langage, paraissaient avoir reçu de l'éducation $»^{42}$.

S'il n'est pas possible de pousser plus avant l'étude de l'usage de ce désignant à la Convention, il est néanmoins intéressant de citer deux exemples de son emploi de la part de girondins, qui, comme on le sait, en donnent une valeur très péjorative en 1793. Les deux citations qui suivent sont du 10 avril, lorsque la droite doit se défendre des attaques des montagnards, et en particulier de Robespierre :

«Qu'entend-on par sans-culottes [s'interroge Pétion] ? Si on entendait par ce mot les braves citoyens du tiers état [...] nous pourrions être avec vous, mais on entend par sans-culottes, non tous les citoyens, les nobles et les aristocrates exceptés, mais tous les hommes qui sont propriétaires pour les distinguer de ceux qui ne le sont pas $»^{43}$.

« A force de crier au peuple qu'il fallait qu'il se levât, dit Vergniaud ; à force de lui parler non pas le langage des lois, mais celui des passions, on a fourni des armes à l'aristocratie ; prenant la livrée et le langage du sans-culottisme, elle a crié dans le département du Finistère : Vous êtes

(41) $A P$, t. 39, p. 281, en note.

(42) $A P$, t. 39 , p. 569 .

(43) Il est clair qu'ici Pétion veut entendre que les « sans-culottes » sont ceux qui ne sont pas des propriétaires. $A P$, t. 61, p. 524 et 556 . 
malheureux, les assignats perdent ; il faut vous lever en masse ! Voilà comme ces exagérations ont nui à la République $»^{44}$.

Cependant, un passage de Brissot de l'été 1792 nous suggère comment, en cette période, la valeur positive de la lexie «sans-culotte(s)» est reconnue aussi par ceux qui la nieront par la suite : « [Ma] section [...] est composée de deux parties ; l'une, respectable, compte un grand nombre de patriotes, ou plutôt de sans-culottes ; l'autre [...] de financiers, d'agents de change, d'agioteurs $»^{45}$.

\section{Des choix discursifs communs. Éléments du « discours montagnard » chez les autres députés}

\section{Monstre(s) ! Le manichéisme discursif dans la description de soi et de l'autre}

Dans l'introduction, nous avons indiqué qu'il y a plusieurs éléments communs au lexique des montagnards et à ceux des autres conventionnels. Pour les approcher, rappelons d'abord que l'un des caractères généralement souligné du discours montagnard, et robespierriste en particulier ${ }^{46}$, est une construction rhétorique fortement manichéenne, qui repose sur l'opposition entre un « peuple », une « nation », une « patrie », des « représentants » décrits comme « bons », « patriotes », « vertueux », etc., et des « factions » « contre-révolutionnaires », « vicieuses », « aristocratiques », « corrompues », « criminelles », etc. Ce genre de construction logique et rhétorique, qu'on retrouve chez les principaux montagnards, est en fait commun à plusieurs députés, et cela notamment dans les moments de crise. Il s'agit ici avant tout d'opposer un « nous » ayant une valeur positive avec un « vous » (voir un «ils ») toujours négatif. En effet, dans ce contexte, le domaine de la morale, de la politique et de la trahison de la patrie et des principes révolutionnaires se mélangent.

(44) Philippe Buchez, Philippe Roux (dir.), Histoire parlementaire de la Révolution française, Paris, Paulin, 1836, t. 25, p. 377. Il n’y a pas le terme « sans-culottisme » dans les AP (t. 61, p. 548).

(45) AP, t. 47, p. 502.

(46) Cf. par exemple, Frank TALlETt, Robespierre and religion, dans Colin HAYDON, William DOYLE (dir.), Robespierre, Cambridge, 1999, p. 103 : « The language that Robespierre adopted when talking of his God [...] there was nothing that was Christian left in his narrow vision of an implacable and unforgiving deity, who presided over a Manichaean universe divided beetween upholders and opponents of the Revolution ». Cf. aussi, par exemple, François FURET, Penser la Révolution française, Paris, Gallimard, 1978 ; Mona OzOUF, « Guerre et Terreur dans le discours révolutionnaire », dans L'école de la France. Essais sur la Révolution, l'utopie et l'enseignement, Paris, Gallimard, 1984, p. 109-127 ; Michel WiNOCK, La grande fracture 1790-1793, Paris, Perrin, 2014. 
Les lemmes positifs qui interviennent dans ce type de discours, interprété selon le prisme des oppositions, sont : «liberté », «égalité », « république », « raison », « nature », « droits naturels », « peuple», « nation », « patrie », « vertu », « bon », « pur », « ami », « solidité », « stoïcisme », etc. Les négatifs sont: «faction», «vice», « crime», « contre-révolution », « aristocratie », «tyrannie », « anarchie », « privilège », « complot », « conspiration », « despotisme », « intrigue », « ambition », « scélératesse », « ennemi », « passions $»^{47}$, « fanatisme », « suspect », « épicurisme », etc. En raison des dynamiques du discours révolutionnaire, à ces derniers s'ajoutent " fédéralisme », « gironde », « modérantisme », « exagération ». Parfois, les lexies négatives sont suivies par des désignants génériques comme " monstre », ou par des lexies liées à l'effusion de sang comme « sangsue », « tigre », « cannibale », « vampire », « buveur de sang », « sanguinaire », « carnage $»^{48}$. Quelques exemples, liés à l'affrontement entre la Gironde et la Montagne, permettront de souligner l'interchangeabilité et la réciprocité d'une partie au moins de ces lexies.

Soulignons d'abord qu' " anarchie », qui est à raison présentée par l'historiographie comme l'une des imputations les plus fréquentes des girondins et des thermidoriens envers les montagnards ${ }^{49}$, est employée par Robespierre le 9 avril 1793 contre ses propres adversaires girondins ${ }^{50}$. Toutefois, elle est aussi utilisée le 5 janvier 1793 lors d'accusations réciproques entre le montagnard Chabot et les girondins Guadet et Salle ${ }^{51}$. Part ailleurs, c'est à travers certains discours des girondins qu'on peut mettre en évidence l'emploi de la construction discursive manichéenne de la part des députés non-montagnards. Un bon exemple en est le discours de Louvet du 29 octobre 1792, dans lequel le conventionnel utilise plusieurs éléments souvent présentés comme étant propres au discours robespierriste : 1) le renvoi fréquent à la vertu, en se référant aux représentants de son propre groupe ( 9 occurrences de «vert*»), qui sont aussi présentés comme les «amis vrais de la liberté », «les

(47) « Passion(s) » ce n'est pas toujours une lexie qui est axiologisée en mal. Il y a, par exemple : «passion de la justice », «passion de l'égalité », «passion de la liberté », etc.

(48) À propos des lexies « aristocratie », « buveur de sang », «fanatisme », « suspect(s)», voir Dictionnaire des usages socio-politiques (1770-1815), fasc. 1, Désignants socio-politiques, Paris, INALF, 1985.

(49) Marc DELEPLACE, «Anarchie/Anarchistes. Germinal-Fructidor an III (21 mars-16 septembre 1795) », dans Dictionnaire des usages socio-politiques (1770-1815), fasc. 4, Désignants socio-politiques, Paris, INALF, 1989, p. 9-33.

(50) Dans le corpus de Robespierre les lexies « anarchie », « anarchiste(s) », apparaissent surtout dans la période de février-avril 1791 et de août 1792 jusqu'à juillet 1793. MU, t. 16, p. 106.

(51) $A P$, t. 56, p. 237 et $s q$ 
plus purs, les meilleurs patriotes »; 2) l'opposition entre cette vertu et la «faction » adverse, décrite comme un repaire d' «ambitieux », de « conjurés », «barbares », « conspirateurs », « factieux » « forcenés », « monstres » ou de « traîtres agitateurs »; 3) la revendication de « l'austère égalité » et de la « liberté publique » comme étant des valeurs propres de son groupe, en opposition à « l'intérêt particulier » des adversaires/ennemis ; 4) l'emploi d'un langage généralement lié au domaine sacré (les lemmes « sacré », « saint », « sacrilège », « impiété ») pour indiquer les valeurs révolutionnaires et ceux qui, aux yeux du locuteur, sont contraires à ces principes.

«Ou je n'ai qu'une fausse idée des mœurs républicaines [dit Louvet], ou la liberté, pure comme la vertu, son inséparable compagne, réprouve ceux qui l'ont servie par des motifs indignes d'elle : et d'ailleurs comment ne pas punir leurs complots, lorsqu'ils en reprennent l'exécrable trame ? [...] Nous, cependant, membres anciens de l'agrégation presque détruite, nous constamment demeurés fidèles aux principes de l'austère égalité, convaincus des mauvais desseins de cette horde de faux-frères conjurés, inquiets de la marche qu'ils comptaient suivre, et nous demandant quels étaient leurs moyens, nous avancions de notre côté dans la carrière révolutionnaire, nous avancions frappant ensemble une cour traîtresse et de traîtres agitateurs [...] nous avancions, bien résolus, quoi qu'il pût arriver, à ne jamais consentir qu'on substituât au saint amour de la patrie l'idolâtrie sacrilège d'un homme [...]. Aussi nous entendîmes bientôt, jusques dans les places publiques, des voix impies réclamant une immense liste où se pressaient entassées des milliers de signatures, la plupart surprises à une aveugle crédulité ; des voix impies qui déjà sollicitaient les biens et le sang de l'innombrable foule des proscrits $»^{52}$.

Un autre exemple est issu d'un discours de Vergniaud, du 13 mars 1793, dans lequel l'auteur attaque une aristocratie générique. De la même manière que Louvet, il se positionne parmi les députés vertueux. Vergniaud, cependant, le fait implicitement, grâce à la description d'une situation hyperboliquement négative :

« Déjà le feu des passions s'est allumé avec fureur dans le sein de cette assemblée, et l'aristocratie ne mettant plus de bornes à ses espérances, a conçu l'infernal projet de détruire la Convention par elle-même ; combinant

(52) $A P$, t. 53, p. 52-58. 
toutes ses démarches d'après le degré d'exaltation des têtes, elle a dit : [...] faisons en sorte que la Convention nationale elle-même soit le cratère brûlant d'où sortent ces expressions sulfureuses, de conspirations, de trahisons, de contre-révolution. Mettons à profit les imprudences d'un patriotisme trop ardent, pour que la colère du peuple paraisse dirigée contre une partie de la Convention par l'autre. Notre rage fera le reste ; et si dans le mouvement que nous aurons excité périssent quelques membres de la Convention, nous présenterons ensuite à la France leurs collègues comme leurs assassins et leurs bourreaux ; l'indignation publique que nous aurons soulevée produira bientôt une seconde catastrophe qui engloutira toute la représentation nationale. D'ailleurs si la Convention absout, l'anarchie régnera et le despotisme viendra au milieu de l'anarchie : alors le tyran qui se cache encore paraîtra sur les débris de sang et de carnage. [...] Souvenezvous qu'il s'agit du salut de la patrie, le moment est venu de prendre un parti décisif. Il faut choisir enfin entre une énergie qui vous sauve et la faiblesse qui perd tous les gouvernements, entre les lois et l'anarchie, entre la république et la tyrannie. $\mathrm{Si}$, ôtant au crime la popularité qu'il a usurpée sur la vertu, vous déployez contre lui une grande vigueur, tout est sauvé. Si vous mollissez, jouets de toutes les factions, victimes de tous les conspirateurs, vous serez bientôt esclaves $»^{53}$.

La construction rhétorique que nous venons de citer, non seulement est employée contre les adversaires politiques strictu sensu (qu'ils soient identifiés personnellement ou vaguement), mais aussi, à la Convention, envers les ennemis sociaux. Cela est principalement mis en œuvre à l'encontre des agioteurs et de ceux qui s'enrichissent grâce aux difficultés d'approvisionnement en matières premières. On peut en citer un exemple d'avril 1793, issu des Observations [...] sur le projet présenté par le comité de Constitution de Sylvain Pepin, député de la Plaine. L'extrait nous rappelle aussi, par son approche «montagnarde » du droit de propriété, que la division de l'Assemblée en Gironde, Plaine et Montagne n'est qu'un schéma général, et que les positions de chaque député varient selon les sujets abordés, bien loin d'une logique stricte de parti :

«La latitude donnée dans cet article au droit de propriété me paraît beaucoup trop grande dans un Etat où tous les citoyens ne sont pas propriétaires, [...] ; elle pourrait devenir funeste à ceux qui n'ont point ou qui ont peu de propriétés ; nous ne pouvons nous dissimuler, en effet, qu'il a existé, 
s'il n'en existe encore, des hommes qui, pour exciter des troubles, et pour anéantir la liberté naissante, ont fait des efforts et même des sacrifices pour augmenter le prix des denrées, pour les faire disparaître ou en empêcher la production ; de pareils monstres ne doivent pas avoir le droit d'abuser de leur propriété ; je désirerais, en conséquence, que l'article fût rédigé en ces termes : "Le droit de propriété consiste en ce que tout homme est le maître de disposer de ses biens, de ses capitaux, de ses revenus et de son industrie, de manière cependant à les rendre utiles pour lui et pour les autres"» ${ }^{54}$.

\section{«L'honorable pauvreté » et l'éloge de la « médiocrité » face aux "riches », aux «bourgeois », aux « marchands » ... « égoüstes ». Des spécificités montagnardes?}

Sans vouloir entrer dans les détails de l'intéressante mais complexe discussion constitutionnelle de 1793 sur le droit de propriété (naturel ou civil, absolu ou limité $)^{55}$, il est cependant important de signaler, dans notre perspective, comment les conventionnels se positionnent par rapport à la richesse et à la pauvreté.

Partons de l'idée que plusieurs montagnards, et sûrement Robespierre, Marat et Saint-Just, postulent une communauté sobre et solidaire, dans laquelle la politique intervient pour réduire les inégalités excessives et assurer à tous un travail, l'instruction, ainsi que des secours face à la maladie et à la vieillesse ${ }^{56}$. Les trois députés en question sont solidaires avec la «pauvreté » et avec le «malheur» et, en général, s'opposent à la richesse et à l'opulence. Ils proposent, comme l'affirme Saint-Just le 23 ventôse an II, un bonheur qui «n'est pas celui des peuples corrompus ; ceux-là se sont trompés, qui attendaient de la Révolution le privilège d'être à leur tour aussi méchants que la noblesse et que les riches de la monarchie ; une charrue, un champ, une chaumière à l'abri du fisc, une famille à l'abri de la lubricité d'un brigand, voilà le bonheur $»^{57}$.

(54) $A P$, t. 63, p. 292-293.

(55) Il serait intéressant de reprendre l'analyse des théorisations sur le droit de propriété dans une optique différente par rapport à l'idée qui veut que la Montagne serait favorable à une limitation du droit civil de propriété, tandis que la Gironde serait favorable à un droit de propriété naturel, individuel et absolu. Sur la pensée économique montagnarde, voir : «Y eut-il une penséee économique de la montagne? », dans Gilbert FACCARELLO et Philippe STEINER (dir.), La pensée économique pendant la Révolution française, Grenoble, PU Grenoble, 1990, p. 211-224.

(56) Voir Jean-Paul MARAT, Projet de déclaration des droits de l'homme et du citoyen, [Paris], 23 août 1789 ; Maximilien ROBESPIERRE, Déclaration des droits de l'homme et du citoyen, dans OMR, t. 5, p. 360-363, en particulier art. XI-XII.

(57) Louis-Antoine SAINT-JUST, Rapport au nom du Comité de salut public sur les factions de l'étranger (23 ventôse an II, 13 mars 1794), Euvres complètes, op. cit., p. 729. 
En revanche, ces hommes ne parlent jamais d'une parfaite égalité sociale et cela est répété à plusieurs reprises lors de l'affrontement avec la Gironde. Le discours de Chabot du 5 mai 1793 illustre ce refus : «Prétendre établir l'égalité sociale, est une absurdité naturelle imaginée par des hommes intéressés à alarmer les propriétaires, parce qu'ils n'ont cessé de tromper les sans-culottes $\gg^{58}$. Pour autant, nous pouvons mettre en évidence que l'éloge de la pauvreté et de la médiocrité n'est pas une prérogative montagnarde, pas plus que les attaques contre l' " opulence », la « richesse » ou les « bourgeois » et « marchands » égoïstes. Nous pouvons notamment l'observer dans le cas du député de la Plaine Azéma, qui est aussi membre du comité de Législation; le 4 juin 1793, a latere de la discussion sur le droit de succession, il se déclare ainsi favorable à la diminution des différences de fortunes les plus frappantes :

« Nous devons travailler à détruire toute espèce de dépendance et d'inégalités, parmi tous les citoyens de la République, qui ont tous juré la liberté, l'égalité et l'unité. Nos lois doivent tendre sans cesse à établir, à ramener et à maintenir la plus grande liberté et la plus parfaite égalité possibles ; [...] il faut travailler sans relâche à augmenter les richesses de l'Etat, en diminuant le nombre des riches et des pauvres. L'homme opulent ne peut l'être que par la misère de son voisin, [...]. Plus nous nivellerons les hommes, plus nous diviserons les fortunes, plus nous les multiplierons, plus nous les accroîtrons, et plus nous soustrairons la misère et l'indigence de la société ${ }^{59}$.

La pauvreté semble donc un sujet généralement (mais pas toujours) axiologisé en bien dans l'Assemblée républicaine, vu que, pour beaucoup, la pauvreté est la « gardienne de la vertu $»^{60}$. L'idée n'est pas étrangère à certains girondins. En effet, même Brissot, en prônant la suspension de la condamnation à mort de Louis XVI, se positionne dans la classe de l' « honorable pauvreté ». Le moins célèbre Bancal des Issarts, quant à lui, en décembre 1792, décrit un électeur-type comme étant « homme vertueux et simple, qui se cache dans l'obscurité de son village et de sa pauvreté $»^{61}$.

(58) $A P$, t. 64, p. 158. Robespierre revient sur ce sujet à plusieurs reprises dans les mêmes termes que Chabot. Voir $O M R$, t. 7, p. 182 et $s q .(5$ avril 1791) ; t. 4, p. 117 (7 juin 1792) ; t. 9, p. 459 (10 mai 1793).

(59) $A P$, t. 66, p. 41.

(60) Maximilien RoBESPIERRE, Discours sur la Constitution (10 mai 1793), dans OMR, t. 9, p. 496.

(61) $A P$, t. 57, p. 406 et t. 55, p. 417 
Moins fréquents sont les cas où la pauvreté est associée au crime, comme chez le député de la Plaine Isidore Wandelaincourt, dans ses Observations sur le plan de Constitution du 24 juin 1793. Il faut cependant souligner qu'ici apparaît la lexie «misère », l'équivalent en négatif de pauvreté : «La misère et la pauvreté, qui ne sauraient dégrader quelques âmes sublimes, sont, dans le commun des hommes, la source des bassesses et des crimes $»^{62}$.

Si donc «pauvreté » est un terme qui peut avoir un caractère ambigu, le véritable idéal social d'une large partie des conventionnels est la «médiocrité ». Il s'agit d'une lexie plus vague que la précédente, qui reprend un idéal générique de modération et de juste milieu. Le girondin Guadet arrive même à se présenter comme son incarnation, le 12 avril $1793^{63}$ :

« Eh bien ! où sont-ils donc ces trésors ? Venez, vous qui m'accusez; venez dans ma maison ([...]) ; venez-y voir ma femme et mes enfants, se nourrissant du pain des pauvres ([...]) ; venez-y voir l'honorable médiocrité au milieu de laquelle nous vivons ; allez dans mon département, voyez-y si mes minces domaines sont accrus ; voyez-moi arriver à l'Assemblée ; y suis-je traîné par des coursiers superbes ?».

Brissot lui même, qui s'était déjà décrit comme étant pauvre, presque à la veille de sa proscription, se défend des attaques en vantant la « médiocrité » de sa condition économique : «Examinez ma vie de tous les jours, suivez-moi dans mon intérieur [...] vous y trouverez l'honnête médiocrité vantée par Horace ${ }^{64}$.

Si la pauvreté et plus encore la médiocrité sont des valeurs généralement louées dans la sphère publique, la contrepartie de cet éloge est souvent la condamnation des « riches », des « bourgeois » et, parfois, des « marchands » en général. Toutefois, après une première et partielle analyse des concordances, il nous semble que, d'une manière générale, la condamnation de la « richesse » est moins répandue que l'apologie de la pauvreté. Néanmoins, ce rejet de l'opulence est plus prononcé chez les montagnards ${ }^{65}$. En effet, on le retrouve souvent chez ces derniers, soit en associant richesse et égoïsme, comme le fait par exemple Danton le 14

(62) $A P$, t. 67, p. 417

(63) $A P$, t. 61, p. 635

(64) $A P$, t. 65, p. 474.

(65) Au moins un exemple issu de la Gironde est celui du discours d'Isnard du 23 février $1793(A P$, t. 59, 123) : «Si l'ennemi triomphe, malheur à ceux qui auront des torts envers la patrie ! Riches, remplissez vos devoirs envers elle, si vous voulez qu'elle soit généreuse envers vous ». 
août 1793 (« et les riches, ces vils égoïstes seraient les premiers la proie de la fureur populaire »), soit par le biais de lexies complexes comme « aristocrate(s) bourgeois ${ }^{66}$, « aristocratie du commerce (Danton) ou autres ${ }^{67}$. Dans plusieurs cas, cette diabolisation de la richesse s'associe, pour les députés de la gauche, à un manque d'esprit de fraternité et/ou d'égalité. Voici comment s'exprime le montagnard Petit le 18 décembre 1792 : « Il est temps de forcer les riches à être les frères des pauvres $»^{68}$.

\section{Le lexique d'une politique économique proprement montagnarde : le cas de la formule « républicaniser le commerce »}

Jusqu'ici nous avons pu mettre en évidence certains éléments communs du discours parlementaire, mais qu'en est-il des spécificités montagnardes ? Pour apporter un début de réponse, nous pouvons nous intéresser à une expression propre au discours montagnard : « républicaniser le commerce ». Cette formule, bien qu'utilisée seulement de février à août 1794, semble être une lexie-vedette très intéressante pour analyser la politique économique des conventionnels à une période pendant laquelle sont notamment adoptées les fameuses lois de ventôse ${ }^{69}$.

La lexie « républicaniser le commerce » apparait, dans les débats de la Convention, le 21 février 1794 ( 3 ventôse an II), dans un rapport de Barère au nom du Comité de salut public ${ }^{70}$. Elle est reprise peu après (le 9 ventôse, 27 février) dans un rapport du jacobin Charles-François Oudot, présenté au nom des comités de Législation, de Commerce et d'Agriculture :

«Il est temps de républicaniser le commerce [...] Il est temps d'apprendre au commerce que ce ne sont plus les richesses qui détermineront la considération publique, mais l'avantage que tire la société d'une profession quelconque ; que, dès que les gains d'un négociant sont immodérés, non-seulement il cesse d'être utile, mais encore qu'il devient un ennemi de la nation par l'usure qu'il fait sur la multitude $»^{71}$.

(66) Amand-Benoît-Joseph Guffroy utilise « aristocrate(s) bourgeois » le 7 janvier 1793 (AP, t. 56, p. 440) ; Robespierre, le 10 avril 1793 (OMR, t. 9, p. 377) ; Barras et Fréron, le 26 juillet 1793 $(A P$, t. 72, p. 556)

(67) $A P$, t. 72 , p. 166 et t. 73 , p. 269

(68) $A P$, t. 55 , p. 135

(69) Les lois du 8 et du 13 ventôse (proposées par Saint-Just) visent à séquestrer les biens des suspects reconnus ennemis de la République et à les distribuer aux patriotes indigents.

(70) $M U$, t. 19 , p. 528.

(71) Ibidem, p. 591 
Dans leurs discours, les deux députés soulignent l'urgence d'une intervention du gouvernement dans la réglementation des échanges, pour éviter l'agiotage. Mais, l'utilisation de la formule « républicaniser le commerce » vise à insister sur la nécessité de changer le système économique, qui doit être guidé par les liens éthiques et fraternels entre les citoyens. Des montagnards illustres comme Robespierre et Saint-Just partagent cette vision de l'économie politique. Toutefois, ils n'utilisent jamais l'expression « républicaniser le commerce », ni le verbe « républicaniser ${ }^{72}$. En revanche, il s'agit d'une lexie qu'ils connaissent sûrement, car « républicaniser le commerce » est utilisé plusieurs fois par Reverchon, représentant en mission à Lyon de la mi-germinal au 17 fructidor an II (des premiers jours d'avril au 3 septembre 1794). Il la prononce dans certaines lettres qu'il écrit à Couthon ${ }^{73}$, dans des discours à la société populaire de Lyon, ainsi que dans un projet économique pour relancer la production de la soie lyonnaise, qu'il soumet au Comité de salut public avec son collègue Jean-Baptiste Dupuy.

Dans ce Mémoire au comité de Salut public sur la réhabilitation du commerce de Commune-Affranchie $e^{74}$, Reverchon et Dupuy proposent d'appliquer les lois de ventôse en donnant à des groupes de deux petits entrepreneurs associés, pauvres mais « patriotes », de l'argent et des matériaux confisqués aux rebelles lyonnais pour leur permettre de commencer la production selon une éthique nouvelle, différente de celle liée exclusivement au profit. En plus, ils proposent de fixer un maximum sur les profits de ces entreprises :

« Pour républicaniser le commerce, il ne s'agit que d'assujettir l'émulation même à un maximum, et de l'arrêter là où commence l'esprit de cupidité $[\ldots]$ nous pouvons aisément diviser cette masse [des fabriques de soie] en trente petits établissemens [...] qui au lieu de procurer en peu d'années une fortune immense à un seul homme, seront un moyen d'aisance pour soixante pères de famille. [...] Nous vous proposons de créer dans cette commune un noyau républicain de commerce et d'industrie, en

(72) Nous n'avons pas retrouvé « républicaniser le commerce » dans le portail ARTFL.

(73) Il s'agit des lettres du 21, 23, 27 et 29 germinal et de la lettre du 7 prairial. Voir Papiers inédits trouvés chez Robespierre, Saint-Just, Payan, etc. supprimés ou omis par Courtois, 3 vol., Paris, Baudouin frères, 1828, t. III, p. 61-75. Les originaux sont conservés aux Archives Nationales, $\mathrm{F}^{7} 4436$ (1).

(74) Jacques REVERCHON, Jean-Baptiste DUPUY, Mémoire au comité de salut public sur la réhabilitation du commerce de Commune-Affranchie, an II, Lyon, Barret, 1834 (dorénavant Mémoire). 
formant trois cents établissemens en faveur de patriotes peu fortunés et capables de les diriger $\gg^{75}$.

Le projet de Reverchon et Dupuy n'évoque pas une économie gérée directement par les structures politiques centrales et locales, mais des aides économiques mises en place par l'Assemblée. Tout en se montrant sensibles aux demandes plus radicales pour un changement dans l'économie de la République, les deux représentants adoptent une position intermédiaire (exprimée à travers la lexie « républicaniser le commerce ») entre une liberté de commerce parfaite et la gestion de la production et des échanges par les structures administratives, demandée par d'autres sujets ${ }^{76}$. En fait, Reverchon et Dupuy soulignent que :

« Il paraît impossible de faire manutentionner le commerce par le gouvernement [...]. Cette idée subversive de toute propriété, aliment [...] de l'industrie et de l'économie, en tuant la liberté des individus, les livrerait [...] à une nouvelle caste d'agens privilégiés qui deviendraient bientôt aussi dangereux et aussi cruels que leurs anciens oppresseurs $»^{77}$.

Trois témoignages nous indiquent que le projet est pris en considération par le Comité de salut public. Le premier est une lettre du Comité de salut public aux deux députés, datée du 7 messidor an II (25 juin 1794) : « Le Comité a particulièrement fixé son attention sur le mémoire [...] qui a pour objet de républicaniser les fabriques de Commune-Affranchie ; il exprimera son opinion à cet égard ». Le deuxième est une affirmation de Collot d'Herbois du $4^{\mathrm{e}}$ sans-culottide an II $^{78}$. Le troisième est une note de Courtois dans son célèbre Rapport fait au nom de la commission chargée de l'examen des papiers de Robespierre (février 1795), dans laquelle il exprime son accord avec les suggestions des deux députés.

À l'extérieur du Comité de salut public, c'est le président du comité du Commerce, François Villiers ${ }^{79}$, qui s'occupe du Mémoire. Il accepte la plupart des propositions de Reverchon et Dupuy, mais souligne, le 12

(75) Mémoire, p. 19.

(76) Par exemple par Buissart, un ami arrageois de Robespierre, qui est avocat et homme de sciences. Lettre de Buissart à Robespierre du 14 pluviôse an II, dans Papiers inédits trouvés, op. cit., t. I, p. 252-253.

(77) Mémoire, p. 13.

(78) Recueil des actes du comité de Salut public, 28 vol., Paris, Imprimerie nationale, 1901, t. 14, p. 522. Les originaux sont aux AN, AFII 37, $M U$, t. 22, p. 17

(79) Soulignons que Villiers comprend bien la nécessité du maximum puisqu'il propose sa prolongation le 21 fructidor an II (7 septembre 1794), contre le nouveau Comité de salut public qui veut le supprimer (AP, t. 96, p. 339). 
messidor (30 juin 1794), que l'acceptation de la logique du maximum est possible seulement à la lumière des « circonstances particulières où se trouve Commune-Affranchie » :

« Il faut y républicaniser le commerce et apprendre aux citoyens qui voudraient s'y donner que c'est l'intérêt général plutôt que celui particulier qui doit les guider dans leurs spéculations. [...] [mais] fixer le nombre de métiers et des ouvriers [...] ne s'accorde pas avec cette liberté qui développe l'industrie. [...] borner l'industrie par des prohibitions, c'est nuire tout à la fois au travail que l'on permet et à celui que l'on défend $»^{80}$.

Toutefois, la chute des robespierristes et les changements politiques qui suivent empêchent l'exécution de ce projet, et entraînent la disparition de la formule « républicaniser le commerce » du lexique politique. Dans les sources que nous avons consultées, elle est prononcée la dernière fois par Reverchon, le 21 thermidor, à la société populaire de Lyon.

En intervenant dans un vaste débat relatif aux choix discursifs et factuels d'un groupe politique, qui ne peut pas être considéré comme un parti au sens moderne du terme, nous avons voulu mettre en évidence trois éléments fondamentaux.

Il s'agissait d'abord de se pencher sur les choix (effectués ou subis) des désignants politiques utilisés par la gauche, par ses adversaires et leurs alliés. Il s'agit d'un système complexe d'hétéro-désignation et d'autodésignation, dans lequel la suprématie politique définit le niveau d'axiologisation de chaque désignant. Pour les trois lexies étudiées (montagnard, girondin, sans-culotte) il semblerait que le point de départ soit toujours une hétéro-désignation (notamment pour les sans-culottes) pour devenir, ensuite, une auto-désignation de réponse. Certains représentants, dont Robespierre, semblent avoir du mal à accepter ces noms jusqu'au moment où ils deviennent d'usage courant.

Le deuxième aspect que nous avons voulu mettre en évidence, c'est l'existence d'une construction discursive commune à toute l'Assemblée, qui est le résultat d'un substrat de valeurs partagées, mais aussi d'une rhétorique qui se nourrit des usages discursifs de l'adversaire. En ce sens, nous avons choisi d'analyser deux catégories du discours : celui basé sur l'opposition nous/vous (ou nous/ils) et l'apologie de la pauvreté/médiocrité.

(80) Fernand Gerbaux, Charles Schmidt (dir.), Procès-verbaux des Comités d'agriculture, 5 vol., Paris, Imprimerie Nationale, 1906-1910, t. 4, p. 394 et $s q$. 
Bien évidemment, nous aurions pu présenter d'autres exemples et valoriser les différences discursives entre les divers groupes, ainsi que celles des membres appartenant au même groupe. Néanmoins, nous avons choisi cette optique, car nous pensons que la mise en évidence des différences a été analysée plus longuement par l'historiographie.

Le troisième élément de notre analyse a mis en relation une introduction lexicale proprement montagnarde avec une politique socio-économique spécifique du groupe. Le projet de «républicaniser le commerce » à Commune-Affranchie et le lexique associé à l'expression peuvent être, de notre point de vue, un bon prisme pour tester les diverses interprétations historiographiques de la Montagne relatives à ses spécificités et à la volonté de modifier la structure de la propriété privée. L'idéal de ce courant était l'indépendance personnelle et un certain degré d'égalité, nécessaires au maintien de la république démocratique qu'ils envisageaient.

Marco MARIN

Università di Trieste Dipartimento di Studi Umanistici Androna Campo Marzio 10 marcomarin77@yahoo.it 